\title{
Erratum to: Implementation of wide-field integration of optic flow for autonomous quadrotor navigation
}

\author{
Joseph Conroy • Gregory Gremillion . \\ Badri Ranganathan $\cdot$ J. Sean Humbert
}

Published online: 12 September 2009

(C) Springer Science+Business Media, LLC 2009

Erratum to: Auton Robot

DOI 10.1007/s10514-009-9140-0

\section{List of corrections}

- Page 2, last paragraph of Sect. 1. Lines 7 through 13 should read: Section 4 discusses the identification of the vehicle dynamics and the implementation of the optic flow-based wide field integration control strategy. Section 5 describes in detail the closed loop stability of the vehicle during corridor navigation. Section 6 presents results and summarizes the effectiveness of wide field integration navigation using optic flow, as observed in the experimentation. Section 7 summarizes the conclusions.

- Page 2. The first paragraph of Sect. 3 should read: In this section a spatially continuous representation of planar optic flow for corridor environments is developed and a model for extracting relative position and orientation based on the $L_{2}$ inner product is introduced.

The online version of the original article can be found under doi:10.1007/s10514-009-9140-0.

J. Conroy $(\varangle) \cdot$ G. Gremillion · B. Ranganathan · J.S. Humbert University of Maryland, 3181 Martin Hall, College Park, MD 20742, USA

e-mail: conroyj@umd.edu

G. Gremillion

e-mail: gmgrem@umd.edu

B. Ranganathan

e-mail: badrinr@umd.edu

J.S. Humbert

e-mail: humbert@umd.edu
- Page 5, last paragraph. Variable $O F_{\text {trans }}$ should be omitted.

- Page 6. The first 3 lines of the first paragraph should read: Given a control gain $K_{v}$, reference height $h_{0}$ and a commanded input $\phi_{r}$, the resulting transfer function for the lateral degree of freedom is

- Page 6. Equation (8) should read:

$$
\frac{v}{\phi_{r}}=\frac{g}{s+\left(Y_{v}+\frac{K_{v}}{h_{0}}\right)} \text {. }
$$

- Page 6. Equation (9) should read:

$$
\dot{r}=-\left(N_{r}+K_{r}\right) r+N_{\mu_{\text {yaw }}} \mu_{\text {yaw }} .
$$

- Page 6. The last sentence of the first paragraph should read: The term $\left(N_{r}+K_{r}\right)$ sets the closed loops bandwidth attained by the vehicle avionics.

- Page 7. Equation (11) should read:

$$
\begin{aligned}
& \ddot{x}=-\left(X_{u}+\frac{K_{u}}{h_{0}}\right) \dot{x}+g \theta_{r}, \\
& \ddot{y}=-\left(Y_{v}+\frac{K_{v}}{h_{0}}\right) \dot{y}+g \phi_{r}, \\
& \ddot{\psi}=-\left(N_{r}+K_{r}\right) \dot{\psi}+N_{\mu_{\text {yaw }}} r_{r} .
\end{aligned}
$$

- Page 7. The last paragraph should read: To close the outer feedback loop that governs heading and lateral control, reference inputs $\phi_{r}$ and $r_{r}$ in (11) are set equal to (12), scaled by gains $K_{y}$ and $K_{\psi}$.

- Page 7. Equation (14) should read:

$$
\begin{aligned}
\phi_{r} & =K_{y}\left\langle\dot{Q}, F_{y}\right\rangle, \\
r_{r} & =K_{\psi}\left\langle\dot{Q}, F_{\psi}\right\rangle .
\end{aligned}
$$

- Page 8. Equation (15) should read: 
$\left\{\begin{array}{c}\dot{y} \\ \ddot{y} \\ \dot{\psi} \\ \ddot{\psi}\end{array}\right\}=\left[\begin{array}{cccc}0 & 1 & 0 & 0 \\ -K_{y} \frac{u_{0} g}{2 a^{2}} & -\left(Y_{v}+\frac{K_{v}}{h_{0}}\right) & 0 & 0 \\ 0 & 0 & 0 & 1 \\ 0 & K_{\psi} \frac{N_{\mu_{\text {yaw }}}}{2 a} & -K_{\psi} \frac{u_{0} N_{\mu_{\text {yaw }}}}{a} & -\left(N_{r}+K_{r}\right)\end{array}\right]\left\{\begin{array}{c}y \\ \dot{y} \\ \psi \\ \dot{\psi}\end{array}\right\}$.

- Page 8, first paragraph. The second sentence should read: The feedback gains $K_{y}$ and $K_{\psi}, \ldots$; and the third sentence should read: ... such that the $K_{y}$ gain was equal to that of the $K_{\psi}$ gain.

- Page 8. Right after the third sentence in Sect. 6 the following text should be inserted: The procedure for each flight test was to lift off and position the vehicle at its initial flight condition at the beginning of the corridor, engage the sonar-based altitude control, input a step in reference pitch angle $\theta_{r}$ to the vehicle to begin moving down the corridor, and engage optic flow for lateral and orientation control. 